\title{
Massive Loss of Mid- and Hindbrain Neurons during Embryonic Development of Homozygous Lurcher Mice
}

\author{
Susie So-Wun Cheng and Nathaniel Heintz \\ Howard Hughes Medical Institute, The Laboratory of Molecular Biology, The Rockefeller University, New York, New York \\ 10021
}

\begin{abstract}
The mouse neurological mutant lurcher $(L c)$ results from a semidominant mutation. Heterozygous $L c /+$ mice are viable but ataxic because $L c /+$ Purkinje cells die by apoptosis within the first 3 weeks of life. $L c / L c$ mice die shortly after birth. To aid in understanding the function of the lurcher gene product, we have examined the embryonic development of homozygous lurcher animals. The ratio of $+/+: L c /+: L c / L c$ animals did not deviate significantly from the expected 1:2:1. Homozygous lurcher mice at PO were found to be normal under gross morphological examination. However, these mice weighed less, lacked milk in their stomach, and died within the first day of life. No resorbed embryos were found at embryonic day (E) 17.5, indicating that all homozygous lurchers survived until birth. Histological examination of PO animals revealed that in homozygous lurcher mice the patterning of the brain is normal but that there has been a massive loss of hindbrain neurons during
\end{abstract}

embryonic development. A particularly conspicuous consequence of the $L c / L c$ genotype at birth is the complete absence of large neurons comprising the trigeminal motor nucleus. These neurons arise normally and are maintained until E15.5. However, beginning at E15.5 large numbers of pyknotic cells are evident in the trigeminal motor nucleus, suggesting that these cells die coincident with their terminal differentiation in the developing hindbrain. Because the trigeminal motor nucleus controls muscles required for suckling, these results suggest an explanation for the neonatal death of homozygous $L c$ animals. These data demonstrate that the severe and dosedependent developmental consequences of lurcher gene action result from degeneration of distinct neuronal populations on maturation in the developing CNS.

Key words: cerebellum; lurcher; homozygous phenotype; cell death; midbrain; hindbrain
The semidominant mouse neurological mutant lurcher was described first by Philips (1960) and later characterized extensively by others (Wetts and Herrup, 1982a,b; Heckroth, 1994a,b; Norman et al., 1995). Heterozygous lurcher mice are fertile, although they have reduced litter size and lower body weight (Wilson, 1975). Adult Lc animals are ataxic and tend to fall in their attempts to walk (Philips, 1960). This results from the complete loss of $L c /+$ cerebellar Purkinje cells beginning during the first few postnatal weeks (Sidman et al., 1965). Studies of $L c \leftrightarrow$ wild-type chimeric mice have shown that Purkinje cell death in $L c /+$ animals is cell-autonomous (Wetts and Herrup, 1982a,b). In both normal and heterozygous lurcher animals, Purkinje cells are born between embryonic days 11 (E11) and 13 (E13) in the developing cerebellar anlage. $L c /+$ Purkinje cells survive in normal numbers beyond birth, although the entire population eventually is lost between postnatal days 8 and 65 (Caddy and Biscoe, 1979). This is followed by the gradual loss of granule cells in the $L c /+$ cerebellum. We recently have shown that $L c /+$ Purkinje cells die by apoptosis and that they begin their final program of differentiation before their death as a consequence of lurcher gene action (Norman et al., 1995).

Despite the extensive studies of the $L c /+$ phenotype and the initial report that lurcher homozygotes probably die shortly after birth (Philips, 1960), very little is known about the homozygous

\footnotetext{
Received Oct. 24, 1996; revised Jan. 9, 1997; accepted Jan. 13, 1997.

This work was supported by the Howard Hughes Medical Institute. We thank John Gubbay and other members of the Heintz Lab for useful discussions.

Correspondence should be addressed to Dr. Nathaniel Heintz, Howard Hughes Medical Institute, The Laboratory of Molecular Biology, The Rockefeller University, 1230 York Avenue, New York, NY 10021.

Copyright (C) 1997 Society for Neuroscience $0270-6474 / 97 / 172400-08 \$ 05.00 / 0$
}

lurcher phenotype. Systematic studies of these animals have been hindered by the lack of reliable genetic markers to genotype accurately the lurcher animals during embryonic development. To gain a better understanding of the action of the $L c$ gene, we have investigated the dose dependence of the $L c$ phenotype in definitively genotyped developing $L c / L c$ animals. We find that survival of homozygous lurcher animals is normal until birth and that all of these animals die within the first day of life. Although gross patterning of the $L c / L c$ brain appears normal, in these animals there is a massive loss of hindbrain neurons during late embryogenesis. In particular, we note as the first sign of the $L c / L c$ genotype the degeneration of the trigeminal motor nucleus, beginning with the death of large neurons in this structure between E15.5 and E16.5 and resulting in its complete absence in P0 pups. Because the trigeminal motor nucleus is required for function of the musculature involved in suckling, loss of this structure contributes to the neonatal death of $L c / L c$ animals. Furthermore, several striking parallels between Purkinje cell death in $L c /+$ animals and motor neuron death in $L c / L c$ animals are evident, suggesting that the dose dependence of the lurcher phenotype results from a differential sensitivity of these neuronal cell types to a single essential factor.

\section{MATERIALS AND METHODS}

Animal crosses and molecular genotyping. Progeny for intercrosses were generated by mating $M$. castaneus with B6CBA-Awj/A- $L c$ mice, purchased from the Jackson Laboratory (Bar Harbor, ME). F1 animals expressing the lurcher phenotype then were selected and intercrossed. Progeny from the F1 intercross can be genotyped after birth as well as at various points in gestation. Genomic DNA was isolated from the tail of mice after birth or from the yolk sac of prenatal mice. Primers MIT-121 and MIT-175 that were found to be mapped closely to lurcher (Zuo et al., 
1995) were used in PCR to amplify the $M$. castaneus allele and B6CBA allele. The PCR product then was resolved on an $8 \%$ acrylamide gel. Mice that are $L c / L c$ have only the B6CBA allele amplified. $L c /+$ will have both the $M$. castaneus and B6CBA alleles amplified, and $+/+$ has only the $M$. castaneus allele amplified.

Isolation of DNA from embryos and neonates. Days 12.5, 15.5, and 16.5 embryos from heterozygous intercrosses were dissected out of uteri, and the yolk sacs were removed for DNA isolation. The DNA was prepared by incubation in tail buffer ( $50 \mathrm{~mm}$ Tris, $\mathrm{pH} 8.0,100 \mathrm{~mm}$ EDTA, and $0.5 \%$ SDS), digestion with proteinase $\mathrm{K}$ overnight at $55^{\circ} \mathrm{C}$, and extraction as described in Hogan et al. (1986). Briefly, the DNA was extracted once with an equal volume of phenol (equilibrated with Tris, $\mathrm{pH} 8.0$ ) and once with phenol/chloroform (1:1); sodium acetate, $\mathrm{pH}$ 6.0, was added to a final concentration of $0.3 \mathrm{M}$. DNA was precipitated with an equal volume of $100 \%$ ethanol, washed in $70 \%$ ethanol, and air-dried. The DNA was resuspended in TE buffer (10 mM Tris, pH 8.0, and $1 \mathrm{~mm}$ EDTA).

Histological examination of paraffin-embedded embryos and PO neonates. Embryos were dissected from decidua at days 12.5, 15.5, and 16.5. Neonates were collected at P0. They were fixed in Bouin's fixative overnight at $4^{\circ} \mathrm{C}$. They were stored in $70 \%$ ethanol and then dehydrated through an ethanol series, cleared in toluene, embedded in paraffin, sectioned at $5 \mu \mathrm{m}$, and stained with hematoxylin and eosin.

Cell Counts. Cells from $\mathrm{P} 0+/+, L c /+$, and $L c / L c$ mice were counted on $50 \mu \mathrm{m}$ sections of brainstem stained with hematoxylin and eosin. Cell counts were made at $400 \times$ magnification on sagittal sections at two locations: in the inferior colliculus and in the pons between the trigeminal motor nucleus and the cerebellum. An objective micrometer and eyepiece reticle were used to calibrate a $6400 \mu \mathrm{m}^{2}$ field for cell counts. Two fields in each of the designated areas on two serial sections from each animal were counted. Cell counts were made from four $+/+$, four $L c /+$, and four $L c / L c$ neonates. Thus, 16 individual fields were counted for each genotype, and the average of these cell counts was expressed as the number of cells per $10,000 \mu \mathrm{m}^{2}$.

\section{RESULTS}

\section{Homozygous lurcher animals survive until birth}

To determine the exact time lurcher homozygotes die, we set up intercrosses between heterozygous lurcher animals that were obtained from the progeny expressing the lurcher phenotype of an intersubspecific cross of B6CBA-Awj/A- $L c$ and $M$. castaneous mice. Using a molecular marker that is tightly linked to the lurcher gene, we were able to genotype accurately the neonates from the intercross. In a total of 83 animals, we obtained a ratio of $21: 44: 18,+/+: L c /+: L c / L c$, in the P0 neonates. This did not deviate significantly from the expected 1:2:1 ratio $(0.9>p>0.5)$, demonstrating that homozygous lurcher animals survive in normal numbers until birth. Furthermore, examination of embryos in two E17.5 pregnant females demonstrated the absence of resorbed decidua in the uterus, as would be expected if $L c / L c$ embryos died in utero. Finally, of the 14 embryos dissected from these pregnant females, all appeared grossly normal. Taken together, these data and the expected ratio of $+/+: L c /+: L c / L c$ demonstrate that the lurcher gene is not essential for embryonic survival.

\section{$L c / L c$ animals are born alive and die within the first postnatal day}

All 83 animals were individually analyzed at P0 using the following criteria. First, we measured the body weight of the P0 animals. Second, we determined their physical status by gross examination. Third, we noted whether there was milk in their stomach. The skin of the $L c / L c$ pups was indistinguishable from that of the wild-type and heterozygote littermates. It was flushed and had a pinkish coloration; there was no abnormality in the respiratory rhythm of $L c / L c$ homozygotes, as compared with their littermates. Thus, soon after birth, their cardiovascular and respiratory systems were functional.

Skeletal preparations were made from some of the $\mathrm{P} 0$ neonates of wild type, $L c /+$, and $L c / L c$ pups. No abnormality was observed in the skeletal structure of any of the neonates. However, the body weights of homozygous lurcher P0 neonates were significantly less than their heterozygous and wild-type littermates. Thus, there was no significant difference in weight between the wild-type and heterozygous neonates $(1.21 \pm 0.18 \mathrm{gm} ; n=16, p>0.1)$, whereas there was a significant reduction in the average weight of $L c / L c$ pups at the time of birth $(1.09 \pm 0.1 \mathrm{gm} ; n=10 ; 0.01>p>$ 0.002 ). Physical examination of the $\mathrm{P} 0$ neonates revealed a class of neonate that was either dead or dying within the first $12 \mathrm{hr}$ after birth. All of these neonates, except one, lacked milk in their stomachs (Fig. 1). This explains the difference in weight between the $L c / L c$ homozygotes and their littermates, because all of these animals were found to be $L c / L c$ when genotyped. We performed autopsies on all of the dead neonates and found that the major organs were present and that they were morphologically normal (data not shown).

\section{Extensive neuron loss in PO Lc/Lc midbrain and hindbrain}

The failure of $L c / L c$ neonates to feed and the cerebellar phenotype of $L c /+$ animals suggested that a possible neuronal deficit in homozygous animals may be responsible for their phenotype. To address this possibility, we undertook an in-depth analysis of the $L c / L c C N S$. P0 neonates were genotyped, fixed in $4 \%$ paraformaldehyde, embedded in paraffin, and sectioned for histochemistry. We first examined the brain in the $L c / L c$ neonates. The gross patterning of the brain appeared normal in $L c / L c$ neonates. Thus, the forebrain, including cortex, hippocampus, thalamus, hypothalamus, and olfactory bulb, appeared to be normal (data not shown), and the general outline of the mid- and hindbrains was not noticeably different from $L c /+$ littermates (data not shown). However, it was readily apparent in $L c / L c$ neonates on close inspection that there was a massive loss of neurons in the midbrain and hindbrain regions of the $L c / L c$ animals. To confirm this finding, we performed cell counts. As shown in Table 3, the average number of cells per $10,000 \mu \mathrm{m}^{2}$ in the midbrain (inferior colliculus) and hindbrain (pons) significantly decreased in neonatal homozygous lurcher animals, whereas the numbers of cells in these areas were not significantly different in the $L c /+$ or $+/+$ littermates. Because the absolute number of cells in the mid- and hindbrains of cerebellar mutant mice is subject to the penetrance of the mutant phenotype on different genetic backgrounds, it is the relative number of cells in $L c / L c$ animals and their sibs that is informative. Thus, there is an $\sim 50 \%$ decrease in cell number in the midbrains of homozygous lurcher animals and an $\sim 40 \%$ decrease in cell number in their hindbrains.

In addition to the general loss of neurons in the mid- and hindbrains of $L c / L c$ pups, we also noted the conspicuous absence of both cerebellar Purkinje cells and of large neurons in the trigeminal motor nucleus. In the case of cerebellar Purkinje cells, this phenotypic characteristic was incompletely penetrant, because some animals clearly contained small numbers of these cells in the developing cerebellum (data not shown). However, in all but one of the $L c / L c$ neonates, it was evident that very few large neurons could be detected in the motor nucleus of the trigeminal nerve (V cranial nerve; Fig. 2). Histological examination of the rest of the remaining body structures, including detailed analysis of all major organs, including the liver, lung, pancreas, heart, and the intestines, did not reveal any abnormalities in $L c / L c$ animals (data not shown). 


\begin{tabular}{|c|c|c|c|c|}
\hline Animal No. & Status at $\mathrm{P} 0$ & Milk in stomach & $\begin{array}{l}\text { Body } \\
\text { weight (gm) }\end{array}$ & Genotype \\
\hline 1 & Dead & No & 1.15 & $L c / L c$ \\
\hline 2 & Weak & No & 1.27 & $L c / L c$ \\
\hline 3 & Good & Yes & 1.35 & N.D. \\
\hline 4 & Good & Yes & 1.34 & $L c /+$ \\
\hline 5 & Good & Yes & 1.00 & $L c / L c$ \\
\hline 6 & Dead & No & 1.07 & $L c / L c$ \\
\hline 7 & Dead & No & 0.99 & $L c / L c$ \\
\hline 8 & Dead & No & 1.02 & $L c / L c$ \\
\hline 9 & Weak & No & 1.06 & $L c / L c$ \\
\hline 10 & Good & Yes & 1.15 & $+/+$ \\
\hline 11 & Good & Yes & 1.16 & $L c /+$ \\
\hline 12 & Good & Yes & 1.39 & $L c /+$ \\
\hline 13 & Good & Yes & 1.04 & $L c /+$ \\
\hline 14 & Good & Yes & 1.22 & $L c /+$ \\
\hline 15 & Good & Yes & 1.39 & $+1+$ \\
\hline 16 & Good & Yes & 1.30 & $+1+$ \\
\hline 17 & Good & Yes & 1.24 & $L c /+$ \\
\hline 18 & Good & Yes & 1.18 & $L c /+$ \\
\hline 19 & Good & Yes & 1.19 & $L c /+$ \\
\hline 20 & Good & Yes & 1.35 & N.D. \\
\hline 21 & Dead & No & N.D. & N.D. \\
\hline 22 & Weak & No & 1.22 & N.D. \\
\hline 23 & Dead & No & 1.08 & $L c / L c$ \\
\hline 24 & Dead & No & 1.15 & $L c / L c$ \\
\hline 25 & Sick & No & 1.06 & $L c / L c$ \\
\hline 26 & Good & Yes & 1.23 & $+1+$ \\
\hline 27 & Good & Yes & 1.28 & $+1+$ \\
\hline 28 & Good & Yes & 1.16 & $L c /+$ \\
\hline 29 & Good & Yes & 1.05 & $L c /+$ \\
\hline 30 & Good & Yes & 1.03 & $+1+$ \\
\hline
\end{tabular}

\section{Degeneration of the trigeminal motor nucleus in LC/Lc embryos}

The reduced number of neurons in the homozygous lurcher mid- and hindbrains, and in particular the absence of large neurons in the trigeminal motor nucleus observed in these animals, was intriguing because axons from this nucleus comprise the $\mathrm{V}$ cranial nerve, which controls the muscles required for mastication and suckling. Because this nucleus is required at birth, these cells undergo synaptogenesis and terminal differentiation during embryonic life. To determine whether neurons in the trigeminal nucleus arise normally during development and then die or whether they are never generated in $L c / L c$ mice, we have studied the development of this structure during embryogenesis.

Embryos from various developmental time points were obtained from timed pregnant females of the heterozygous intercross to allow definitive genotypic characterization. Because the trigeminal nerve can be identified as early as E10, we followed the development of the motor nucleus at E12.5, E15.5, E16.5, and P0. As shown in Figure 3, histological examination of homozygous lurcher embryos at E12.5 revealed that patterning of the developing brain is normal. Although the brain of the $L c / L c$ embryo shown in this figure appears smaller than that of its $L c /+$ littermate, this was not a consistent finding. Thus, no significant differences in the gross anatomy of the $L c / L c$ versus $L c /+$ embryos were evident. In particular, examination of the hindbrains of E12.5 $L c / L c$ embryos revealed that the trigeminal motor was present in the nucleus at this age (Fig. 4). The large motor neurons of this nucleus appear indistinguishable in $L c / L c$ animals when compared with their $L c /+$ and wild-type (not shown) littermates. Furthermore, there was no large difference in neuronal cell number in either the hindbrain or midbrain at this age. These results establish that the lurcher gene does not perturb the generation of neurons in this region of the developing brain. Inspection of sections from E15.5 $L c / L c$ embryos (Fig. 5) reveals that this brain region remains essentially normal between E12.5 and E15.5, although an occasional pyknotic neuron can be noticed in the $L c / L c$ animals.

In contrast, examination of E16.5 embryos revealed a dramatic difference between $L c / L c$ homozygotes and their littermates. As shown in Figure 6, an obvious increase in the number of pyknotic motor neurons was evident in E16.5 $L c / L c$ animals. In these sections, a majority of motor neurons in the trigeminal nucleus are dying, as indicated by their pyknotic profiles. The morphology of these cells as they die, particularly the obvious nuclear condensation and pyknosis, strongly suggests that they are dying by apoptosis. At this age, there is still no gross difference in neuronal number outside of the trigeminal motor nucleus, indicating that death of the large motor neurons occurs first, followed by a general loss of mid- and hindbrain neurons between E15.5 and 


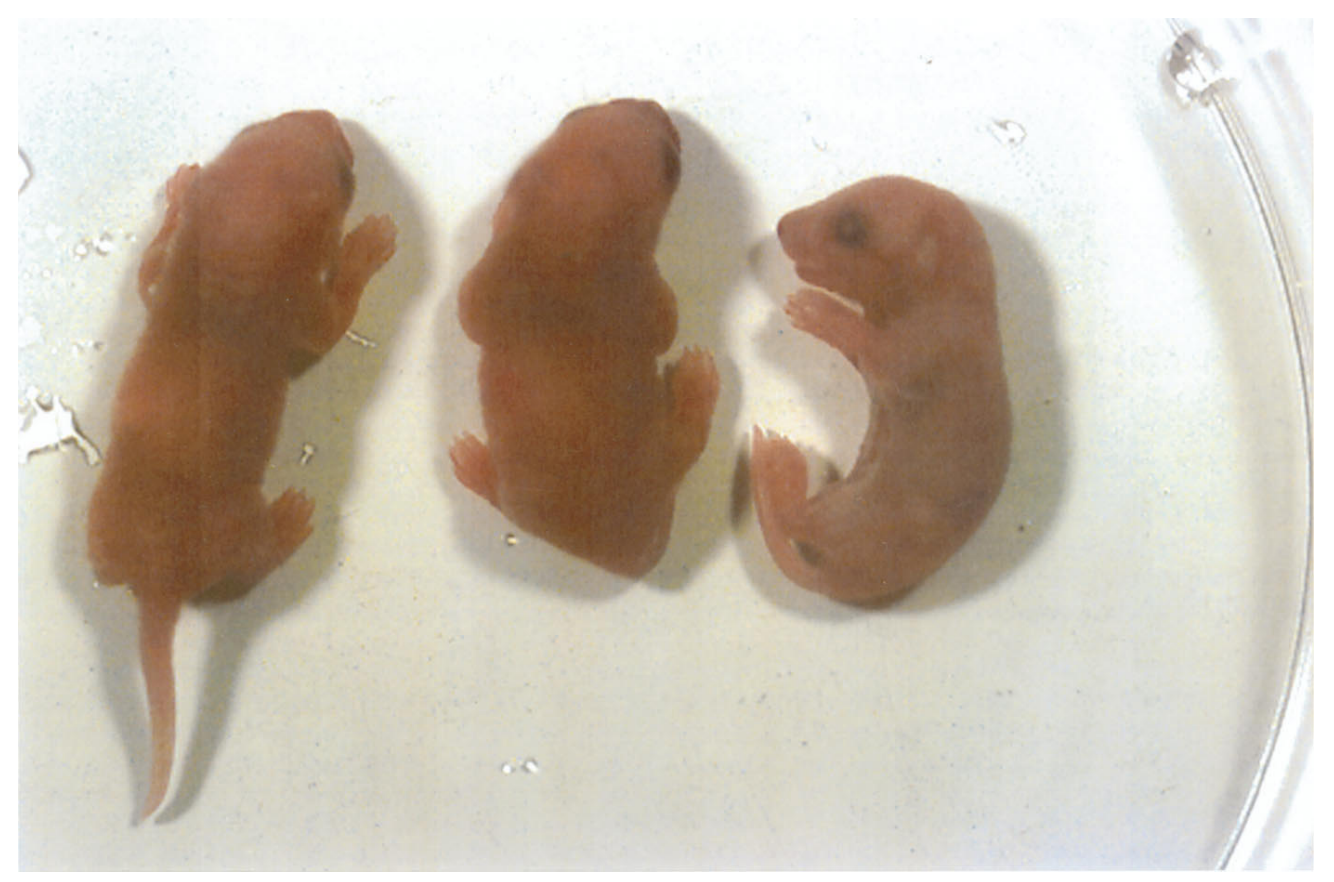

Figure 1. $\quad \mathrm{P} 0$, Newborn animals from $L c /+$ intercross. Left, $+/+$; center, $L c /+$; right, $L c / L c$.

\begin{tabular}{llll}
\hline Table 2. Genotype of P0 neonates from $L \boldsymbol{c} /+\boldsymbol{M}$. Castaneous intercross & & \\
$L c /+\times L c /+$ & $+/+$ & $L c /+$ & $L c / L c$ \\
\hline Observed & 21 & 44 & 18 \\
Expected & 20.75 & 41.5 & 20.75 \\
\hline
\end{tabular}

Table 3. Neuron counts per $10,000 \mu \mathrm{m}^{2}$ of midbrain and hindbrain regions of $+/+, L c /+$, and $L c / L c$ P0 neonates

\begin{tabular}{lcl} 
& Midbrain $400 \times$ & Hindbrain $400 \times$ \\
\hline$+/+$ & $98.4 \pm 11.7$ & $49.4 \pm 4.3$ \\
$L c /+$ & $106.2 \pm 8.9$ & $48.2 \pm 5.4$ \\
$L c / L c$ & $50.0 \pm 4.9$ & $33.1 \pm 4.5$ \\
\hline
\end{tabular}

birth. These data clearly establish that in homozygous lurcher animals the trigeminal motor nucleus degenerates after its formation in the developing brain.

\section{DISCUSSION}

To gain additional insight into the functions of the mouse lurcher gene, we have analyzed the homozygous lurcher phenotype in definitively genotyped lurcher embryos and neonates. In this study, we report several results that are directly relevant to the semi-dominance of lurcher gene action. First, homozygous lurcher animals do not die in utero but fail to feed after birth and die within the first postnatal day. Second, at birth the homozygous lurcher mid- and hindbrains, in contrast to other regions of the CNS, display a strongly reduced neuronal density and a conspicuous absence of large neurons comprising the trigeminal motor nucleus. Third, the loss of these neurons is attributable to neuronal degeneration rather than to a developmental perturbation that results in failure to generate them at the appropriate time. This is consistent with the observation that the gross patterning of the $\mathrm{CNS}$ in developing homozygous lurcher animals appears normal.
Finally, the first neurons to die in the developing hindbrain of $L c / L c$ animals are the motor neurons of the trigeminal motor nucleus. This is followed by a general loss of neurons in this region of the developing brain. It should be noted that it remains possible that homozygous lurcher embryos display earlier, more subtle phenotypic effects. However, because of the genetic heterogeneity required to genotype definitively these animals, evaluation of such subtle differences between homozygous and heterozygous lurcher embryos is problematic. For this reason, highly refined studies of $L c / L c$ gene action must await identification of the lurcher mutation and analysis of its consequences in inbred mouse strains.

The abnormalities that we have observed in the mid- and hindbrain regions of homozygous lurcher animals suggest a possible explanation for the neonatal death of homozygous lurcher animals. The trigeminal motor nucleus provides efferent axons for the $\mathrm{V}$ cranial nerve that innervate muscles from the first branchial arch, which are primarily responsible for movements of the jaw involved in suckling and mastication. Therefore, degeneration of this structure in $L c / L c$ embryos can explain both the lack of milk in the stomachs of homozygous animals after birth and their lower weight within the first 12-24 hr of life. Although we think that the loss of this motor nucleus could be sufficient to explain the neonatal lethality of the homozygous lurcher mutation, the general loss of neurons throughout the mid- and hindbrain regions may be a contributing factor, because animals starved for the first postnatal day sometimes can survive (Klein et al., 1993).

The neuronal degeneration that we have documented in the brainstem of homozygous $L c$ animals is reminiscent of the neuronal loss that has been documented previously in the cerebellum of lurcher 

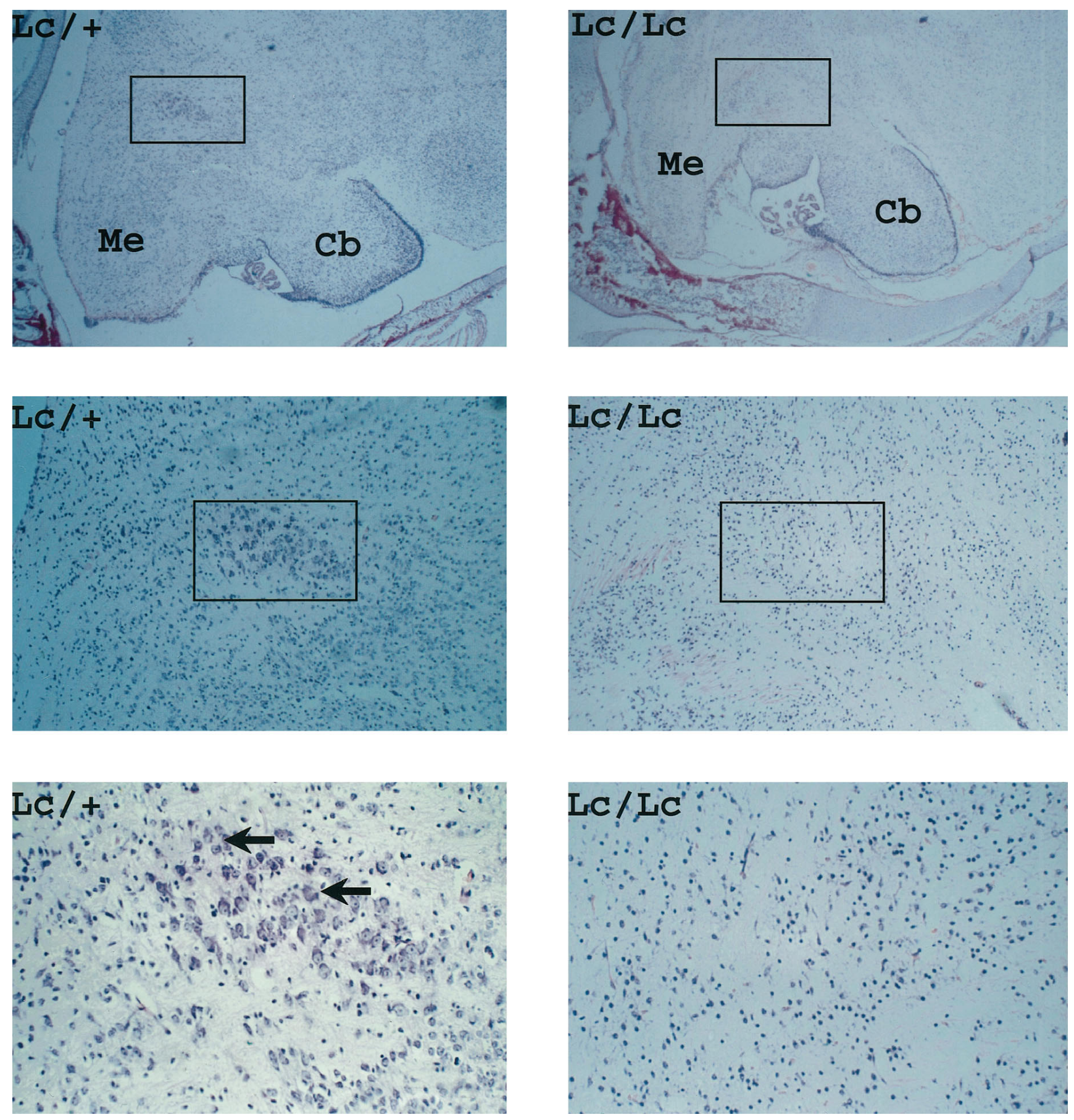

Figure 2. P0, Paraffin sections of neonatal brains from $L c /+$ intercross. Top panels, A low magnification view showing patterning of mid- and hindbrains at birth. At higher magnification (middle panels) the decreased cellular density within the hindbrain of $L c / L c$ embryos is evident. Bottom panels, At high magnification, the motor nuclei of $\mathrm{V}$ cranial nerve from $L c /+$ mice and its absence in equivalent area of the brain in $L c / L c$ animals. $M e$, Medulla; $C b$, cerebellum. Arrows point to trigeminal motor neurons. Magnification: $A, B, 6 \times ; C, D, 20 \times ; E, F, 400 \times$.

heterozygotes (Sidman et al., 1965). In $L c /+$ animals, cerebellar degeneration is initiated at the time of Purkinje cell terminal differentiation as they become integrated into mature cerebellar circuits. This degeneration begins at $\sim \mathrm{P} 10$ with the initial massive death of Purkinje cells and is followed by the gradual disappearance of cerebellar granule cells and inferior olivary neurons. Purkinje cell death in heterozygous animals has been shown to be cell-autonomous (Wetts and Herrup, 1982a,b) and to be dependent on terminal differentiation of these large, primary cerebellar neurons (Messer et al., 1991; Norman et al., 1995). In homozygous lurcher animals, degeneration of the mid- and hindbrain neurons begins with massive death of large neurons in the trigeminal motor nucleus at $\sim$ E15.5E16.5. The timing of these deaths correlates with terminal differentiation of these neurons and maturation of this motor circuitry. Motor neuron death in the trigeminal nucleus is followed by a gradual loss of mid- and hindbrain neurons, perhaps as a secondary 

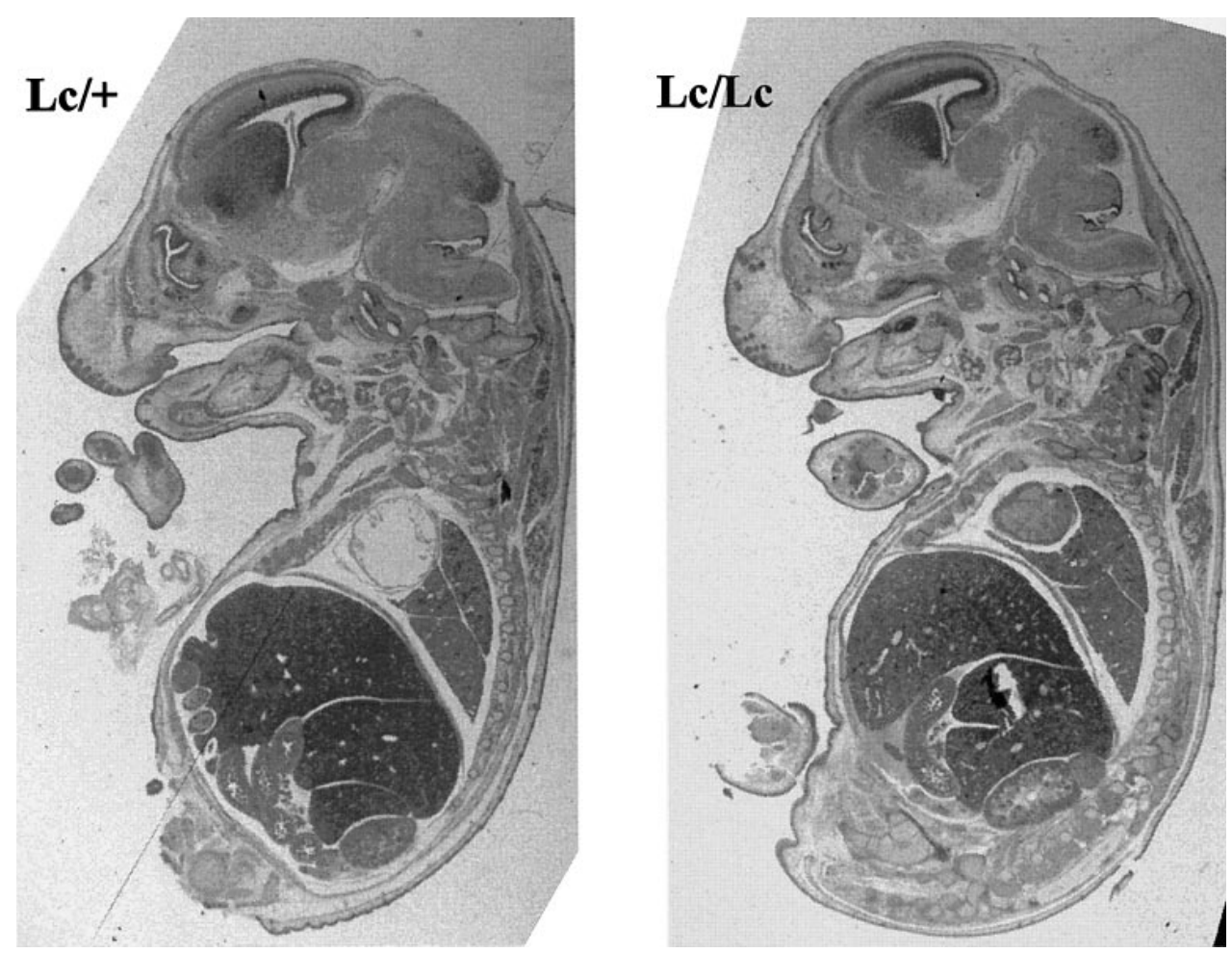

Figure 3. Paraffin sections of E12.5 embryos from the $L c /+$ intercross. Low magnification views of sagittal sections of whole E12.5 $L c /+$ and $L c / L c$ embryos revealed no differences in gross anatomy. In particular, patterning of the $L c / L c$ brain appears normal.
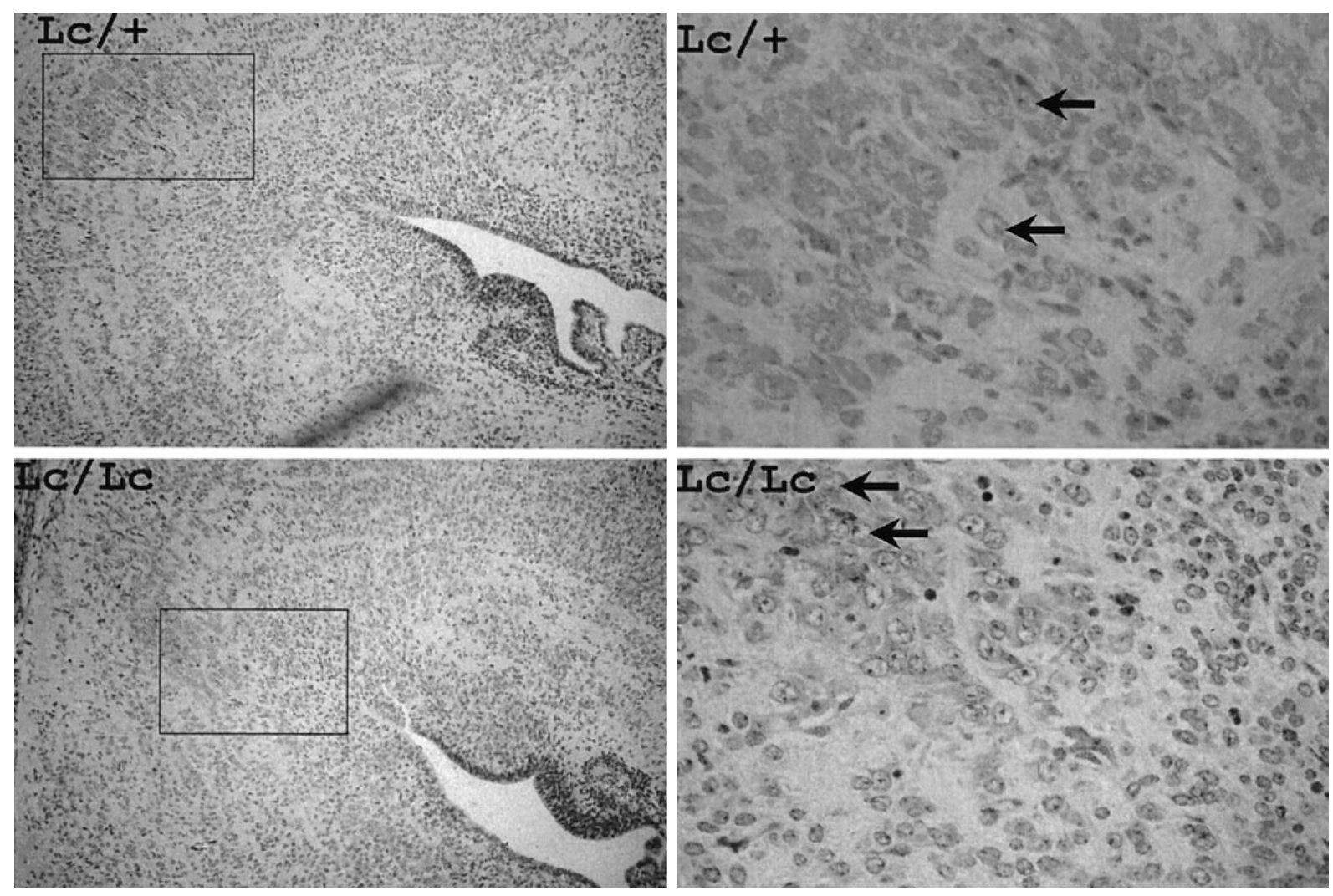

Figure 4. Paraffin sections of E12.5 brains from $L c /+$ intercross. Left panels present low magnification views of the hindbrain region containing trigeminal motor nucleus (outlined) in $L c / L c$ and $L c /+$ animals. Higher magnification views showing trigeminal motor neurons (arrows) are shown in the right panels. Magnification: $A, B, 20 \times ; C, D, 400 \times$. 

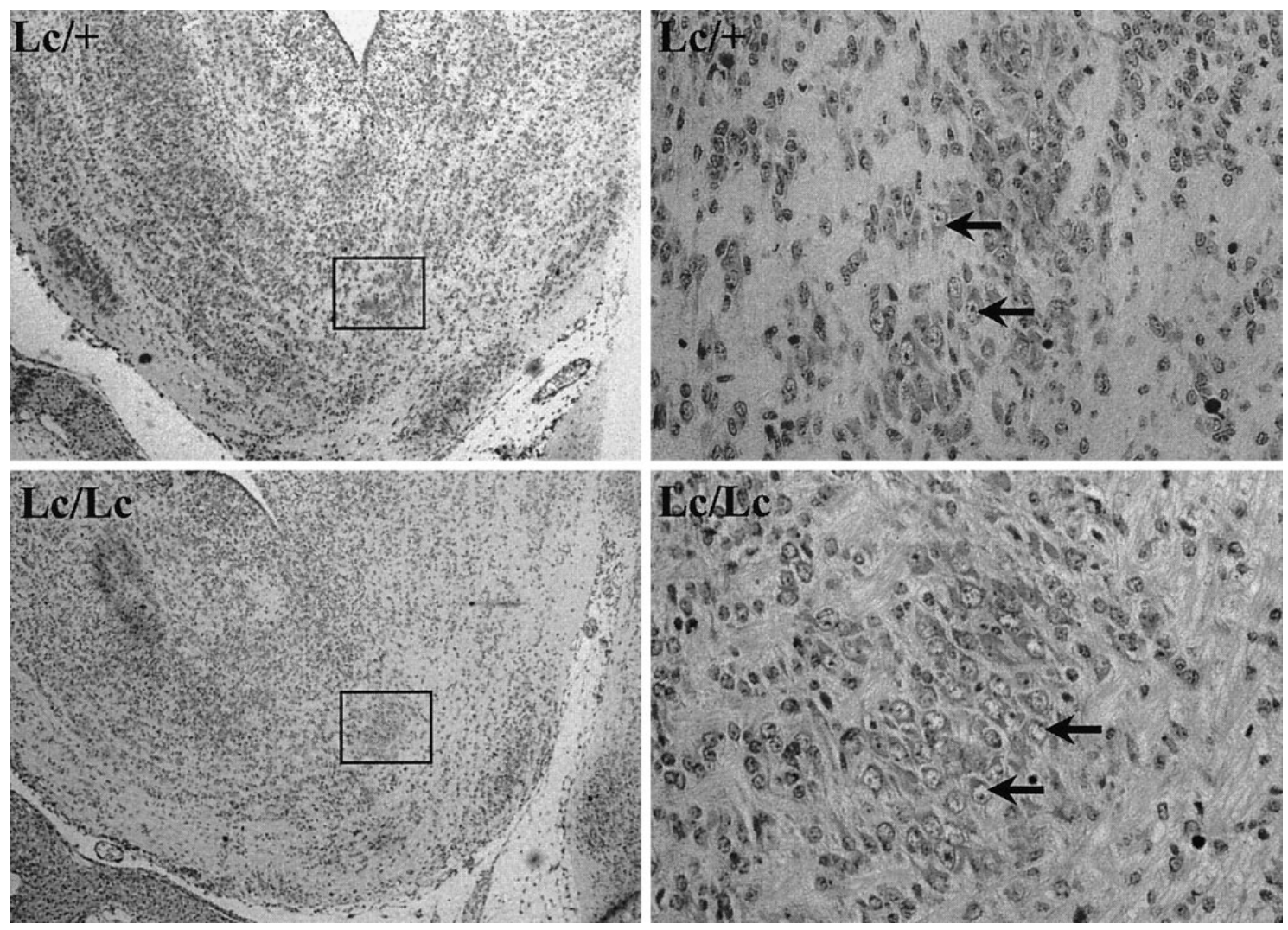

Figure 5. Paraffin sections of E15.5 brains from $L c /+$ intercross. Low magnification views of the hindbrain region containing trigeminal motor nucleus (outlined) are presented in the left panels. Higher magnification pictures showing trigeminal motor neurons (arrows) are presented in the right panels. Magnification: $A, B, 20 \times ; C, D, 400 \times$.
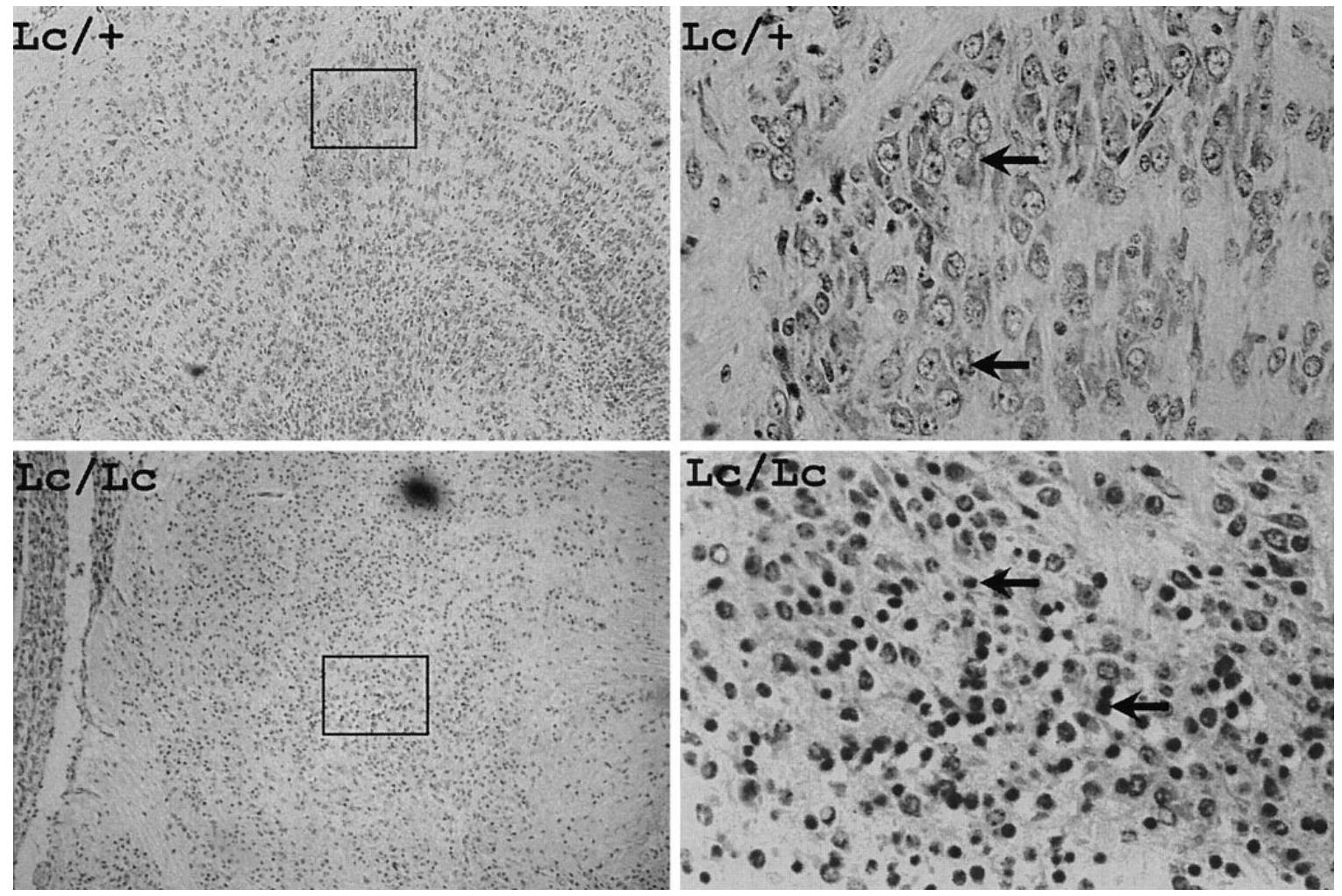

Figure 6. Paraffin sections of E16.5 brains from $L c /+$ intercross. Low magnification views of the hindbrain region containing trigeminal motor nucleus (outlined) are shown at the left. Higher magnification views showing trigeminal motor neurons (arrows) in $L c /+$ animals and a large number of pyknotic profiles (arrows) in $L c / L c$ animals are shown on the right. Magnification: $A, B, 20 \times ; C, D, 400 \times$. 
consequence of the mutation. Thus, these two neuronal populations display a dose-dependent requirement for an intrinsic factor that is essential for viability.

What might this factor be? Although an answer to this question necessarily must await the identification of the $L c$ gene, the results of this analysis provide important clues to its biological roles. Thus, it is clear from the timing and properties of Purkinje cell death in $L c /+$ animals (Norman et al., 1995) and the present data demonstrating trigeminal motor neuron death in $L c / L c$ animals commencing between E15.5 and E16.5 that the lurcher gene acts as these cells become integrated into their respective motor circuits. In both of these cases, lurcher gene action impacts on the survival of differentiated neurons well past the epoch of naturally occurring cell death that is characteristic of the development of these cell types in normal embryos. One might envisage two distinct classes of mechanisms to explain the lurcher phenotype. Thus, it is well established that role of neurotrophins in CNS neurons is complex, influencing both the survival of neurons during critical early developmental periods and later during maturation of these neurons into CNS circuitry. It is thought that these survival functions may be supplied by different neurotrophins at different stages of neuronal differentiation (for review, see Barde, 1989) (Davies, 1988; Snider, 1994). One might imagine, therefore, that the timing of neuronal death in response to the lurcher mutation may reflect a failure of Purkinje cells and trigeminal motor neurons to receive trophic support at this critical final stage in their development. Failure of a pathway involved in trophic support of these neurons also would explain the dose dependence of the lurcher phenotype, because survival in response to trophic factors in vivo and in vitro has been demonstrated to be dose-dependent (Henderson et al., 1993). The fact that lurcher Purkinje cells die by apoptosis is also consistent with the idea that these cells fail to receive appropriate trophic support. Finally, the degeneration of the trigeminal motor nucleus in $L c / L c$ animals, their neonatal death, and their failure to feed are very similar to the phenotype displayed by null mutants for the trkB neurotrophic factor receptor (Klein et al., 1993). Given these considerations, it seems reasonable to postulate that the lurcher gene may encode a product, the function of which is important in mediating trophic factor action in specific CNS neurons.

Alternatively, it seems possible that the semidominant character of the lurcher gene results from a mutation that directly can activate programmed cell death inappropriately as these two cell populations terminally differentiate in the developing brain. According to this model (Heintz et al., 1993), the induction of the apoptotic program in postmitotic neurons is a normal physiologic response to failures in cellular homeostasis that occur as a direct consequence of abnormal gene action. One might imagine that the lurcher mutation results in ectopic or inappropriate activation of signal transduction events in cerebellar Purkinje cells and trigeminal motor neurons that are of sufficient magnitude in the context of normal neuronal activity to elicit programmed cell death. In this case, it is not the failure on trophic support that results in neuronal cell death. Rather, the semidominance of lurcher gene action is a direct consequence of aberrant signaling events caused by the lurcher gene product that act in a dosedependent manner to activate the apoptotic pathway. Given the contrasts between these two models, it will be fascinating to decipher the molecular events involved in dose-dependent activation of cell death in each of these neuronal populations, once the lurcher gene has been identified.

\section{REFERENCES}

Barde YA (1988) What, if anything, is a neurotrophic factor? Trends Neurosci 11:343-351.

Caddy KW, Briscoe TJ (1979) Structural and quantitative studies on the normal C3H and lurcher mutant mouse. Philos Trans R Soc Lond [Biol] 287:167-201.

Davies AM (1988) Role of neurotrophic factors in development. Trends Genet 4:139-143.

Heckroth JA (1994a) Quantitative morphological analysis of the cerebellar nuclei in normal and lurcher mutant mice. I. Morphology and cell number. J Comp Neurol 343:173-182.

Heckroth JA (1994b) A quantitative morphological analysis of the cerebellar nuclei in normal and lurcher mutant mice. II. Volumetric changes in cytological components. J Comp Neurol 343:183-192.

Heintz N (1993) Cell death and the cell cycle: a relationship between transformation and neurodegeneration. Trends Biochem Sci 18:157-159.

Heintz N, Norman DJ, Gao W-Q, Hatten ME (1993) Neurogenic approaches to mammalian brain development. In: Genome analysis, Vol 6, Genome maps and neurological disorders, pp 19-44. Cold Spring Harbor, NY: Cold Spring Harbor Laboratory.

Henderson CE, Camu W, Mettling C, Gouin A, Poulsen K, Karihaloo M, Rullamas J, Evans T, McMahon SB, Armanini MP (1993) Neurotrophins promote motor neuron survival and are present in embryonic limb bud. Nature 363:266-267.

Hogan B, Constantini F, Lacy E (1986) Manipulating the mouse embryo. Cold Spring Harbor, NY: Cold Spring Harbor Laboratory.

Klein R, Smeyne RJ, Wurst W, Long LK, Auerbach BA, Joyner AL, Barbacid M (1993) Targeted disruption of the trkB neurotrophin receptor gene results in nervous system lesions and neonatal death. Cell 75:113-122.

Messer A, Eisenberg B, Plummer J (1991) The lurcher cerebellar mutant phenotype is not expressed on a staggerer mutant background. J Neurosci 11:2295-2302.

Norman DJ, Feng L, Cheng SS, Gubbay J, Heintz N (1995) The lurcher gene induces apoptotic death in cerebellar Purkinje cells. Development (Camb) 121:1183-1193.

Phillips RJS (1960) "Lurcher," a new gene in linkage group XI of the house mouse. J Genet 57:35-42.

Sidman RL, Green MC, Appel SH (1965) Catalogue of the neurological mutants of the mouse. Cambridge, MA: Harvard UP.

Snider W (1994) Functions of the neurotrophins during nervous system development: what the knock-outs are teaching us. Cell 77:627-638.

Wetts RKH, Herrup K (1982a) Interaction of granule, Purkinje, and inferior olivary neurons in lurcher chimeric mice. I. Qualitative studies. J Embryol Exp Morphol 68:87-98.

Wetts RKH, Herrup K (1982b) Interaction of granule, Purkinje, and inferior olivary neurons in lurcher chimeric mice. II. Granule cell death. Brain Res 250:358-362.

Wilson DB (1975) Brain abnormalities in the lurcher $(L c)$ mutant mouse. Experientia (Basel) 31:220-221.

Zuo J, De Jager P, Heintz N (1995) Generation of a high resolution genetic map and a YAC contig of the lurcher locus on mouse chromosome 6. Genome Res 5:381-392. 